\title{
Pineal Transplants in oculo: Limitations on the Ability of Collateral Sprouts of Foreign Neurons to Establish Normal Function
}

\author{
Jaisri R. Lingappa and Richard E. Zigmond \\ Department of Pharmacology, Harvard Medical School, Boston, Massachusetts 02115
}

The pineal gland is innervated by sympathetic neurons whose cell bodies are located in the superior cervical ganglia. This pathway, which carries information concerning environmental lighting to the gland, is important for maintaining a circadian rhythm in the activity of the pineal enzyme serotonin $\mathrm{N}$-acetyltransferase (NAT). Lesioning this pathway blocks the normal nighttime increase in NAT activity. Following transplantation of the pineal gland to the anterior chamber of the eye, the gland becomes reinnervated by collateral sprouts of sympathetic neurons that innervate the iris. In addition, a day-night rhythm in NAT activity is restored to the transplanted glands (Moore, 1975; Bäckström et al., 1976).

These findings raise the possibility that the neural regulation of pineal function may be restored to normal by collateral sprouts of foreign neurons. To determine whether this is the case, other aspects of the neural regulation of NAT activity were examined in transplanted and in situ pineal glands. When the dark period was extended into the normal light period, NAT activity decreased to daytime values in both groups, suggesting that, in both cases, darkness is only effective in maintaining high levels of NAT activity at certain times during the day-night cycle. In contrast to their similar responses to extended darkness, the 2 groups of pineal glands responded differently to a brief pulse of light during the dark period. While the light exposure caused a dramatic decrease in nighttime NAT activity in in situ pineal glands, it produced no change in this enzyme activity in transplanted glands. On the other hand, propranolol, a $\beta$-adrenergic antagonist, caused a rapid decrease in enzyme activity in both groups of pineal glands, indicating that, as in situ pineal glands, transplanted pineal glands exhibit a rapid decrease in NAT activity upon cessation of adrenergic stimulation. These findings indicate that collateral sprouting by foreign sympathetic neurons can establish certain, but not all, aspects of normal pineal function and suggest that the neural information carried by "iris" and "pineal" neurons is not functionally equivalent.

In certain areas of the central and peripheral nervous systems, collateral sprouting of intact ncurons occurs following a lesion. It has been proposed that, in some instances, this phenomenon

\footnotetext{
Received July 14, 1986; revised Apr. 27, 1987; accepted May 5, 1987.

This research was supported by NIH Grant NS 17512. J.R.L. is a trainee of the U.S. Public Health Service (Grants 5T32GM07226 and 5T32GM07306) and R.E.Z. is a recipient of an NIMH Research Scientist Development Award (MH00162). l-Propranolol was a gift from Ayerst Laboratories, New York, N.Y. We would like to thank Dr. Chauncey W. Bowers for helpful comments on the manuscript.

Correspondence should be addressed to Richard E. Zigmond, Department of Pharmacology, Harvard Medical School, 250 Longwood Ave., Boston, MA 02115. Copyright (C) 1987 Society for Neuroscience $0270-6474 / 87 / 113525-04 \$ 02.00 / 0$
}

may be involved in recovery of normal function (Moore, 1980). One technique that has proven useful for studying collateral sprouting is the transplantation of tissues to the anterior chamber of the eye. When an adrenergically innervated tissue is transplanted to the anterior chamber, it becomes reinnervated by collateral sprouts from sympathetic neurons that innervate the iris. Within 2-3 weeks after transplantation, the adrenergic innervation of the tissue appears qualitatively normal by catecholamine fluorescence histochemistry (Olson and Malmfors, 1970). In certain cases (e.g., smooth muscle, heart, cerebellum, and hippocampus), electrical stimulation of the reinnervating neurons produces appropriate responses in the transplanted tissue, indicating that the collateral sprouts have formed effective connections with the target cells (Olson and Malmfors, 1970; Olson and Seiger, 1976; Taylor et al., 1978). In the case of pineal gland transplants, a neurally mediated day-night rhythm in the activity of the enzyme serotonin $N$-acetyltransferase (NAT), similar to the rhythm found in in situ pineal glands, is established following innervation of the transplant (Moore, 1975; Bäckström et al., 1976). NAT activity is important in regulating the rate of synthesis of the pineal hormone melatonin (Klein and Weller, 1970). Since the rhythm in NAT activity in unoperated animals appears to be dependent on a rhythm in the activity of the sympathetic nerves that innervate the gland (Klein et al., 1971; Bowers and Zigmond, 1980, 1982), the transplantation studies raise the possibility that collateral sprouts from nonpineal neurons are capable of establishing normal physiological control over the transplanted tissue. In this paper, we investigate this suggestion further by examining certain additional features of the neural regulation of pineal NAT activity in in situ and transplanted pineal glands.

One characteristic of the regulation of the pineal gland is that exposure of animals to darkness does not produce an increase in NAT activity unless the dark exposure coincides with the "sensitive" phase (the dark phase) of the endogenous circadian rhythm (Binkley et al., 1973; Alphs and Heller, 1978). In this report, we compare the effect on NAT activity, in both in situ and transplanted pineal glands, of extending the dark period by $2 \mathrm{hr}$ into the normal light period (i.e., into the insensitive phase). A second characteristic of the neural regulation of the pineal gland is that when rats are exposed to a light pulse in the middle of the dark period, NAT activity decreases rapidly and remains low for the rest of the dark period (Klein and Weller, 1972; Illnerová et al., 1979). It has been hypothesized that such a pulse of light acts by way of the CNS to cause a phase-shift in the central oscillator that controls the circadian rhythm (Illnerová and Vanecek, 1979, 1982; Zatz and Brownstein, 1983). This change is thought to lead to an abrupt decrease in the firing of the sympathetic neurons that innervate the pineal gland, resulting in a rapid inactivation of NAT molecules and a cessation 
of NAT synthesis. In this paper, we compare the responses of transplanted and in situ pineal glands to a brief light pulse given in the middle of the dark period. A preliminary report of these studies has been presented (Lingappa and Zigmond, 1984).

\section{Materials and Methods}

Unoperated animals and transplant recipients were male Sprague-Dawley rats $(100-125 \mathrm{gm})$ that were housed individually on a $12 \mathrm{hr} / 12 \mathrm{hr}$ light/dark cycle and given food and water ad libitum for $10 \mathrm{~d}$ after their arrival from the supplier (Charles River Breeding Laboratories, Wilmington, MA). Unless specified otherwise, donor pineal glands were frorn 1-d-old Sprague-Dawley rats that arrived from the supplier on the day of operation. Neonates were decapitated, and their pineal glands were removed and kept in $0.9 \% \mathrm{NaCl}$ for a few minutes. Before removal of the donor pineal glands, recipient rats were anesthetized with chloral hydrate, s.c. $(735 \mathrm{mg} / \mathrm{kg})$. A scalpel blade was used to puncture the cornea of recipients, and iridectomy scissors were used to make the puncture into a small incision. Donor pineal glands were picked up with forceps by the pineal stalk and placed inside the anterior chamber on the iris. Two weeks postoperatively, the eyes of the recipient rats were examined under a dissecting microscope to determine whether the transplant had become vascularized. Rats that exhibited heavy scarring of the lens or the cornea, signs of intraocular bleeding, or poorly placed transplants were removed from the experiment at that time. Rats with successful transplants were killed by decapitation 4-6 weeks after transplantation. Olson and Malmfors (1970) found that during this period of time an iris transplanted to the anterior chamber of the eye becomes innervated by adrenergic neurons to $90-105 \%$ of control values, as judged by fluorescence histochemistry.

For determination of NAT activity, rats were decapitated 7-8 hr into the dark period under dim red light (Kodak Safelight filter 2, $15 \mathrm{~W}$ bulb). We have previously found NAT activity to be maximal between 7 and $11 \mathrm{hr}$ into the dark period (Bowers and Zigmond, 1980). The pineal glands of unoperated animals were removed within $1 \mathrm{~min}$ of decapitation, frozen on dry ice, and subsequently stored at $-80^{\circ} \mathrm{C}$. In the case of animals with transplants, the transplanted glands were removed first, followed by removal of the in situ glands, with the entire procedure taking a total of $5 \mathrm{~min}$. One group of rats was anesthetized with chloral hydrate during the light period 4 weeks after transplantalion, and their cervical sympathetic trunks were cut bilaterally $5 \mathrm{~mm}$ caudal to the superior cervical ganglia. One week later, peak night NAT activity was determined. When the effects of a brief light pulse were tested, animals were exposed to $1 \mathrm{~min}$ of light $7-8 \mathrm{hr}$ into the dark period, returned immediately to the dark for $1 \mathrm{hr}$, and then decapitated under dim red light. In one experiment, rats were injected subcutaneously $6-8 \mathrm{hr}$ into the dark (under dim red light) with $l$-propranolol- $\mathrm{HCl}$ $(10 \mathrm{mg} / \mathrm{kg})$ or vehicle alone $(0.9 \% \mathrm{NaCl})$ and were decapitated $20 \mathrm{~min}$ later. In the extended darkness experiment, rats were kept in darkness for $2 \mathrm{hr}$ beyond the time when the dark period normally ended.

Pineal NAT activity was measured according to the method of Deguchi and Axelrod (1972a), as modified by Parfitt et al. (1975). Details of the assay conditions have been reported previously (Bowers and Zigmond, 1980). Protein was measured by the method of Lowry et al. (1951), with bovine serum albumin as the standard. All data are expressed as mean values \pm SEM. The NAT activities of different groups were compared using Student's $t$ test for 2 means (2-tailed). In early experiments, occasional transplants in all groups had no detectable NAT activity. This may have been due to failure of sympathetic innervation of these glands. These transplants are included in the data presented here; however, the observed differences between groups remain significant even when these "failures" are excluded from the calculations.

\section{Results}

The effect of prolonging the dark period into the normal daytime was examined in unoperated rats. NAT activity decreased by $99 \%$ from peak night values when animals were exposed to 2 $\mathrm{hr}$ of extra darkness (data not shown). When the same experiment was carried out using rats with pineal transplants, NAT activity decreased by $99 \%$ in the in situ pineal glands $(p<0.001)$, and by $93 \%$ in the transplanted glands $(p<0.001)$ (Fig. 1).

The effect of a brief light pulse on peak night NAT activity was examined in unoperated rats. When the rats were exposed

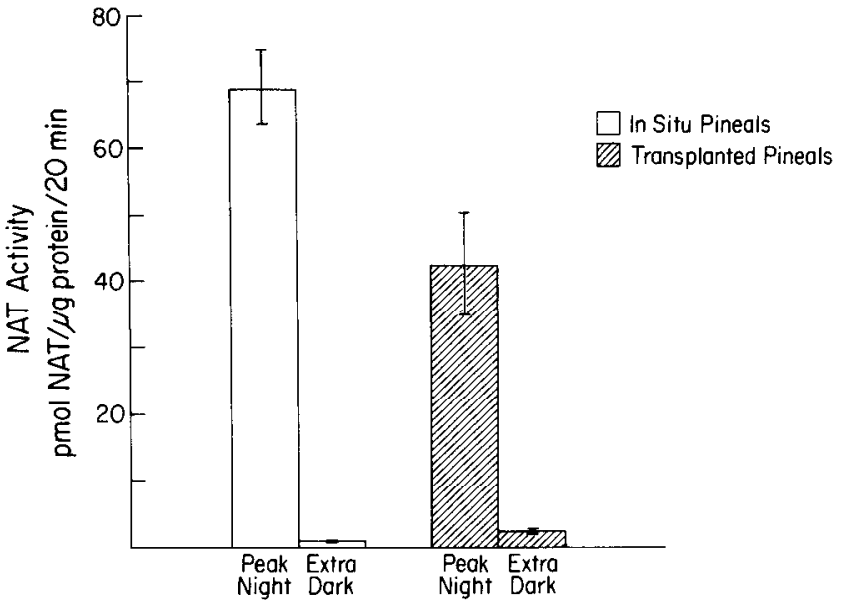

Figure 1. Effects of extending the dark period on NAT activity in in situ and transplanted pineal glands. Four weeks after transplantation, NAT activity was measured in in situ and transplanted pineal glands 7-8 hr into the dark period (Peak Night) and after the $12 \mathrm{hr}$ dark period was extended by 2 more hr (Extra Dark). Each bar represents the mean of $9-10$ pineal glands.

to $1 \mathrm{~min}$ of light 7-8 hr into the dark period and then returned to a dark room for $1 \mathrm{hr}$, NAT activity decreased by $98 \%$, compared to that of animals left in the dark $(p<0.001$; data not shown), as previously reported by Illnerová et al. (1979). When the same experiment was performed using rats with pineal transplants, NAT activity decreased in the in situ pineal glands by $98 \%(p<0.001)$; however, enzyme activity in the transplanted glands did not change significantly (Fig. 2). Similar results were obtained in a second experiment, in which the transplanted pineal glands were taken from adult, rather than neonatal, donors (data not shown).

The failure of NAT activity in the transplanted glands to decrease following a light pulse could indicate that the high nocturnal NAT activity in these glands is not dependent on sympathetic neuronal activity. To examine this possibility, enzyme activity was measured in a group of animals whose cervical sympathetic trunks had been cut bilaterally 1 week earlier. In this group (BiCSTX in Fig. 2) there was a $99 \%$ drop in peak night NAT activity in the in situ pineal glands $(p<0.001)$ and an $88 \%$ drop in activity in the transplanted glands $(p<0.01)$. These results demonstrate that peak night NAT activity in the transplants, like that in the in situ pineal glands, is largely dependent on peripheral sympathetic innervation, thus confirming the findings of Moore (1975).

It is possible that a light pulse causes cessation of sympathetic neural stimulation of both in situ and transplanted pineal glands, but that only in situ pineal glands respond with a rapid decrease in NAT activity. To determine whether this could be the case, the effect of rapidly blocking adrenergic stimulation of the glands was examined by injecting the $\beta$-adrenergic antagonist propranolol. Animals were injected with propranolol or saline in the middle of the dark period and NAT activity was examined 20 min later (Fig. 3). As expected, propranolol caused a 95\% decrease in NAT activity in the in situ pineal glands $(p<0.001)$. Propranolol also caused a similar decrease in NAT activity in the transplanted glands $(p<0.005)$.

\section{Discussion}

The activity of pineal NAT appears to reflect the extent of sympathetic stimulation of the gland. For example, the normal 


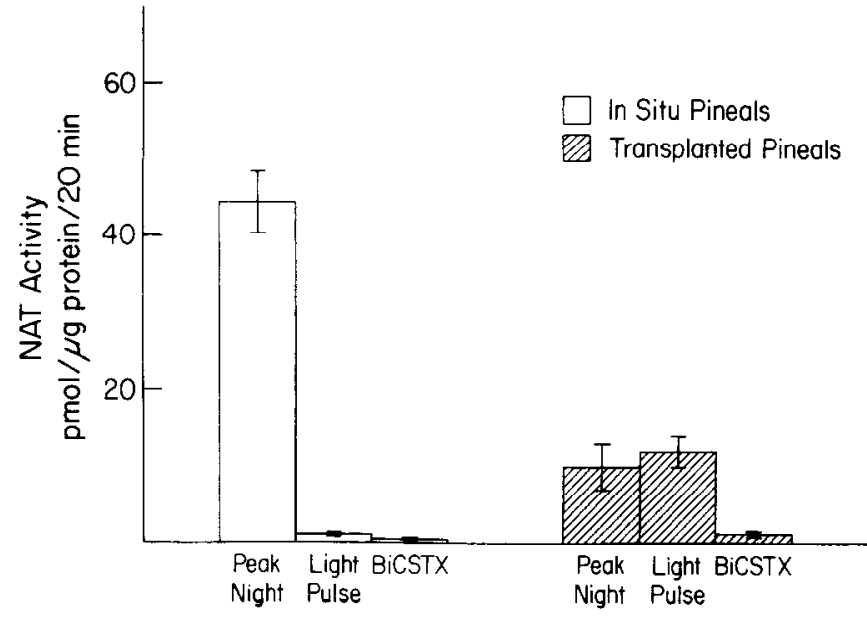

Figure 2. Effects of a brief light pulse and of cutting the cervical sympathetic trunks on the nocturnal NAT activity of in situ and transplanted pineal glands. Five weeks after transplantation of a neonatal pineal gland to the anterior chamber of the eye, NAT activity was measured $7-8 \mathrm{hr}$ into the dark period in in situ (open bars) and transplanted (hatched bars) pineal glands under control conditions (Peak Night), after 1 min of light followed by an hour of darkness (Light Pulse), and 1 week after bilateral cutting of the cervical sympathetic trunks (BiCSTX). Each bar represents the mean of 10-12 pineal glands. [The NAT activity present in the transplants following preganglionic nerve section, though low, was still significantly greater than that found in the in situ pineal glands of the same animals $(p<0.05)$. This difference may reflect a greater stimulation of the transplants by blood-borne catecholamines, due perhaps to prejunctional or postjunctional supersensitivity (Lingappa, 1985). A similar explanation may underlie the small but significant difference in NAT activity between in situ and transplanted pineal glands exposed to a prolonged dark period (see Fig. 1).]

nighttime increase in NAT activity can be prevented by cutting the internal carotid nerves, which contain the axons of neurons in the superior cervical ganglia that innervate the pineal gland (Zigmond et al., 1981; Bowers et al., 1984), by cutting the preganglionic input to the superior cervical ganglia (Klein et al., 1971 ) or by injecting animals with propranolol, a $\beta$-adrenergic antagonist (Deguchi and Axelrod, 1972b). In addition, low daytime NAT activity can be increased by injection of the $\beta$-adrenergic agonist isoproterenol (Deguchi and Axelrod, 1972b) or by electrical stimulation of the preganglionic cervical sympathetic trunks (Volkman and Heller, 1971; Bowers and 7igmond, 1980, 1982). These results and others suggest that the sympathetic neurons innervating the pineal gland fire more rapidly at night than during the day. Furthermore, the rapid decrease in nighttime pineal NAT activity following a brief light pulse appears to reflect a decrease in the activity of these neurons (IIlnerová and Vanecek, 1979; Bowers and Zigmond, 1980). A similar rapid decrease in enzyme activity is seen following cessation of electrical stimulation of the cervical sympathetic trunks (Bowers and Zigmond, 1980).

The findings of a day-night rhythm in NAT activity in pineal transplants could indicate that collateral sprouts of the sympathetic neurons that innervate the iris establish normal neural control over the pineal glands. Such an interpretation would imply that the neural information carried by "pineal" and "iris" neurons is functionally equivalent. This idea is supported by the finding that, when darkness is extended into the expected light phase, nocturnal NAT activity in both in situ and transplanted pineal glands decreases to daytime values. Such a decrease in NAT activity during the daytime, even in the absence

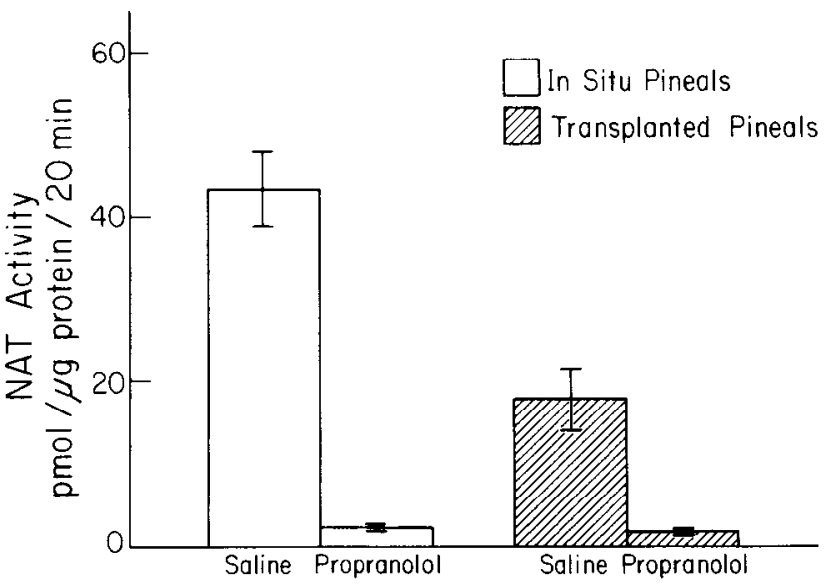

Figure 3. Effects of $l$-propranolol on nighttime NAT activity in in situ and transplanted pineal glands. Six weeks after transplantation, NAT activity in in situ (open bars) and transplanted (hatched bars) pineal glands was measured 6-8 $\mathrm{hr}$ into the dark period, 20 min after a subcutaneous injection of $l$-propranolol $(10 \mathrm{mg} / \mathrm{kg})$ or $0.9 \% \mathrm{NaCl}$. Each bar represents the mean of 7-10 pineal glands. [Note that peak night NAT activity of transplanted pineal glands ranged in different experiments from 23 to $62 \%$ of the values of in situ pineal glands (Figs. 13).]

of environmental lighting cues, is consistent with the idea that in both groups of pineal glands the day-night rhythm in NAT activity is generated by an endogenous circadian pacemaker. That the rhythm in NAT activity is circadian has been established for pineal glands of unoperated animals by demonstrating that a rhythm in NAT activity persists in blinded animals and in animals kept in constant darkness (Klein and Weller, 1970). Similar experiments would be needed to provide definitive evidence that the rhythm in the transplanted glands is also circadian. Nevertheless, the data here suggest that the sympathetic neurons that innervate both in situ and transplanted pineal glands fail to fire at normal nighttime levels when darkness coincides with the endogenous light period.

Thus, the neural regulation of in situ and transplanted pineal glands seems to share a number of features. With both, it appears that nerve activity is higher at night than during the day, and that this activity is under the control of an endogenous circadian pacemaker. In electrical stimulation studies, it has been found that NAT activity decreases very rapidly upon cessation of sympathetic nerve stimulation (Bowers and Zigmond, 1980). Thus, the observation of a day-night rhythm in the transplanted pineal glands suggests that this high rate of firing is sustained during the night in iris neurons, as it is in pineal neurons.

However, despite the commonalities discussed above, our results suggest that significant differences exist between the populations of sympathetic neurons that normally innervate the pineal gland and those that normally innervate the iris. An hour after a brief light pulse, peak night NAT activity in transplanted pineal glands is unchanged, while peak night NAT activity in in situ pineal glands is dramatically decreased. That pineal neurons mediate a light-induced inhibition of NAT activity, while iris neurons do not, suggests that iris neurons and pineal neurons carry functionally different information from the CNS. The possibility that the transplanted pineal glands are simply incapable of undergoing a rapid decrease in NAT activity upon cessation of $\beta$-adrenergic stimulation is ruled out by the demonstration that propranolol causes a rapid decrease in nighttime NAT ac- 
tivity in both transplanted and in situ pineal glands. A second possible explanation is that high NAT activity in the transplants is maintained following the light pulse by a nonsympathetic neural input that innervates the transplants but does not innervate the in situ glands. However, this explanation is unlikely, since a bilateral lesion of the cervical sympathetic trunks eliminates most of the nighttime NAT activity in both groups of pineal glands.

Previous studies have demonstrated that innervation by collateral sprouts of foreign neurons can reestablish many aspects of the integrity of a transplanted tissue (i.e., restoration of organotypic innervation, of appropriate synaptic transmission upon electrical stimulation, and of some functional characteristics of the transplanted tissue) (Hoffer et al., 1975; Moore, 1975; Seiger and Olson, 1975; Bäckström et al., 1976). However, such innervation may not be sufficient to establish all normal tissue functions. Interestingly, restoration of certain functions and not others has also been observed in the case of transplants of fetal substantia nigra into the brains of animals whose nigrostriatal pathway has been lesioned unilaterally. One explanation that has been proposed for this selectivity is that different functions are subserved by different regions of the striatum and, depending on where the foreign substantia nigra neurons are placed, only certain functions will be restored (Dunnet et al., 1983). In contrast, in the situation presented in the current study, we suggest that selective establishment of function may result from the particular frequency and pattern of nerve activity of the innervating neurons. Different populations of neurons may share certain similarities in the neuronal information they carry (by way of the same or different central circuits)-for example, a day-night rhythm in firing-while, at the same time, having functionally relevant differences in this information. As a result, following innervation by collateral sprouts of foreign neurons, some functional characteristics may appear while others may not. It is important, then, to use a wide range of tests to assess the extent to which normal function has been established.

\section{References}

Alphs, L., and A. Heller (1978) A circadian rhythm in dark induction of rat pineal serotonin: Coenzyme A : $N$-acetyltransferase activity. Brain Res. 139: 374-377.

Bäckström, M., L. Olson, and Å. Seiger (1976) $N$-Acetyltransferase and hydroxyindole- $O$-methyltransferase activity in intraocular pineal transplants: Diurnal rhythm as evidence for a functional sympathetic adrenergic innervation. Acta Physiol. Scand. 96: 64-71.

Binkley, S. A., D. C. Klein, and J. L. Weller (1973) Dark induced increase in pineal serotonin $\mathrm{N}$-acetyltransferase activity: A refractory period. Experientia 29: 1339-1340.

Bowers, C. W., and R. E. Zigmond (1980) Electrical stimulation of the cervical sympathetic trunks mimics the effects of darkness on the activity of serotonin: $N$-acetyltransferase in the rat pineal. Brain Res. 185: 435-440.

Bowers, C. W., and R. E. Zigmond (1982) The influence of the frequency and pattern of sympathetic nerve activity on serotonin $N$-acetyltransferase in the rat pineal gland. J. Physiol. (Lond.) 330 : 279-296.

Bowers, C. W., L. M. Dahm, and R. E. Zigmond (1984) The number and distribution of sympathetic neurons that innervate the rat pineal gland. Neuroscience 13: 87-96.

Deguchi, T., and J. Axelrod (1972a) Sensitive assay for serotonin $N$-acetyltransferase activity in rat pineal. Anal. Biochem. 50:174179.

Deguchi, T., and J. Axelrod (1972b) Control of circadian change of serotonin $\mathrm{N}$-acetyltransferase activity in the pineal organ by the $\beta$-adrenergic receptor. Proc. Natl. Acad. Sci. USA 69: 2547-2550.

Dunnett, S. B., A. Björklund, R. H. Schmidt, U. Stenevi, and S. D. Iversen (1983) Intracerebral grafting of neuronal cell suspensions. IV. Behavioural recovery in rats with unilateral 6-OHDA lesions following implantation of nigral cell suspensions in different forebrain sites. Acta Physiol. Scand. (Suppl.) 522: 29-37.

Hoffer, B., L. Olson, A. Seiger, and F. Bloom (1975) Formation of a functional adrenergic input to intraocular cerebellar grafts: Ingrowth of inhibitory sympathetic fibers. J. Neurobiol. 6: 565-585.

Illnerová, H., and J. Vanecek (1979) Response of rat pineal serotonin $\mathrm{N}$-acetyltransferase to one min light pulse at different night times. Brain Res. 167: 431-434.

Illnerová, H., and J. Vanecek (1982) Two oscillator structure of the pacemaker controlling the circadian rhythm of $\mathrm{N}$-acetyltransferase in the rat pineal gland. J. Comp. Physiol. 145: 539-548.

Illnerová, H., J. Vanecek, J. Krecek, L. Wetterberg, and J. Saaf (1979) Effect of one minute exposure to light at night on rat pineal serotonin $\mathrm{N}$-acetyltransferase and melatonin. J. Neurochem. 32: 673-675.

Klein, D. C., and J. L. Weller (1970) Indole metabolism in the pineal gland: A circadian rhythm in $N$-acetyltransferase. Science 169: 10931095.

Klein, D. C., and J. L. Weller (1972) Rapid light-induced decrease in pineal serotonin $N$-acetyltransferase activity. Science 177: 532-533.

Klein, D. C., J. L. Weller, and R. Y. Moore (1971) Melatonin metabolism: Neural regulation of pineal serotonin:acetyl coenzyme A $N$-acetyltransferase activity. Proc. Natl. Acad. Sci. USA $68: 3107$ 3110.

Lingappa, J. R. (1985) Recovery of function and changes in innervation of the pineal gland following regeneration and collateral sprouting of sympathetic neurons. Ph.D. thesis, Harvard University, Cambridge, MA.

Lingappa, J. R., and R. E. Zigmond (1984) A comparison of the regulation of serotonin $N$-acetyltransferase (NAT) activity in normal pineal glands and pineal glands transplanted in oculo. J. Steroid Biochem. 20: 1457.

Lowry, O. H., N. J. Rosenbrough, A. L. Farr, and R. J. Randall (1951) Protein measurement with the Folin phenol reagent. J. Biol. Chem. 193: 265-275.

Moore, R. Y. (1975) Pineal transplants to the anterior chamber of the eye: Evidence for functional reinnervation. Exp. Neurol. 49:617-621.

Moore, R. Y. (1980) Regeneration in the mammalian nervous system. Ann. NY Acad. Sci. 339: 102-114.

Olson, L., and T. Malmfors (1970) Growth characteristics of adrenergic nerves in the adult rat. Fluorescence histochemical and $\mathrm{H}^{3}$ noradrenaline uptake studies using tissue transplants to the anterior chamber of the eye. Acta Physiol. Scand. (Suppl.) 348: 1-112.

Olson, L., and $\AA$. Seiger (1976) Beating intraocular hearts: Lightcontrolled rate by autonomic innervation from host iris. J. Neurobiol. 7: 193-203.

Parfitt, A., J. L. Weller, K. Sakai, B. H. Marks, and D. C. Klein (1975) Blockade by ouabain or elevated potassium inn concentration of the adrenergic and adenosine cyclic $3^{\prime}, 5^{\prime}$-monophosphate-induced stimulation of pineal serotonin $N$-acetyltransferase. Mol. Pharmacol. 11 . 241-255.

Seiger, Å., and L. Olson (1975) Brain tissue transplantation to the anterior chamber of the eye: 3 . Substitution of lacking central noradrenaline input by host iris sympathetic fibers in the isolated cerebral cortex developing in oculo. Cell Tissue Res. 159: 325-338.

Taylor, D., A. Seiger, R. Freedman, L. Olson, and B. Hoffer (1978) Electrophysiological analysis of reinnervation of transplants in the anterior chamber of the eye by the autonomic ground plexus of the iris. Proc. Natl. Acad. Sci. USA 75: 1009-1012.

Volkman, P. H., and A. Heller (1971) Pineal $N$-acetyltransferase activity: Effect of sympathetic stimulation. Science 173: 839-840.

Zatz, M., and M. J. Brownstein (1983) Injection of $\alpha$-bungarotoxin near the SCN blocks the effects of light on nocturnal pineal enzyme activity. Brain Res. 213: 438-442.

Zigmond, R. E., C. Baldwin, and C. W. Bowers (1981) Rapid recovery of function after partial denervation of the rat pineal gland suggests a novel mechanism for neural plasticity. Proc. Natl. Acad. Sci. USA 78: 3959-3963. 